\title{
Characterizing 3D Shapes Using Fractal Dimension
}

\author{
André Ricardo Backes ${ }^{1}$, Danilo Medeiros Eler ${ }^{2}$, \\ Rosane Minghim ${ }^{2}$, and Odemir Martinez Bruno ${ }^{3}$ \\ ${ }^{1}$ Faculdade de Computação - Universidade Federal de Uberlândia \\ Uberlândia MG Brasil \\ backes@facom.ufu.br \\ 2 Instituto de Ciências Matemáticas e de Computação (ICMC) \\ Universidade de São Paulo (USP) \\ Avenida do Trabalhador São-carlense, 400 \\ 13560-970 São Carlos SP Brazil \\ danilome@gmail.com, rminghim@icmc.usp.br \\ 3 Instituto de Física de São Carlos (IFSC) \\ Universidade de São Paulo (USP) \\ Avenida do Trabalhador São-carlense, 400 \\ 13560-970 São Carlos SP Brazil \\ bruno@ifsc.usp.br
}

\begin{abstract}
Developments in techniques for modeling and digitizing have made the use of 3D models popular to a large number of new applications. With the diffusion and spreading of $3 \mathrm{D}$ models employment, the demand for efficient search and retrieval methods is high. Researchers have dedicated effort to investigate and overcome the problem of $3 \mathrm{D}$ shape retrieval. In this work, we propose a new way to employ shape complexity analysis methods, such as the fractal dimension, to perform the 3D shape characterization for those purposes. This approach is described and experimental results are performed on a 3D models data set. We also compare the technique to two other known methods for 3D model description, reported in literature, namely shape histograms and shape distributions. The technique presented here has performed considerably better than any of the others in the experiments.
\end{abstract}

Keywords: Fractal dimension, complexity, 3D shape descriptor.

\section{Introduction}

The use of 3D models is growing on the Internet and in specific domains (e.g., Biology, Medicine and Archaeology). This fact is directly related to new acquisition technologies, such as 3D scanners, and to new 3D graphics rendering technologies; these in turn are also related to the evolution of graphics hardware, CPUs and modeling tools, that have eased the construction of $3 \mathrm{D}$ models $[12$.

This growth requires new efficient mechanisms to organize, search and retrieve these $3 \mathrm{D}$ models from large repositories. To solve that problem, efforts 
have been made to investigate the specific problem of 3D shape retrieval. Which has been solved by effective description methods. Generally, 3D shape retrieval approaches are based on feature methods, which calculate geometric and topological properties from 3D objects; graph methods, which extract a graph representing geometric meaning from shape components; and other methods (e.g., based on 3D appearance, shape deformation and view similarity) 3244 .

In this work, we propose to use shape complexity analysis methods, such as the fractal dimension, to perform 3D shape characterization. Fractal dimension is described in the literature as a non-integer value related to the complexity of a Fractal object (non-Euclidean geometry). In shape analysis, this property enables us to quantify the shape complexity in terms of space occupation and self-similarity [56/78]. We also compare this complexity analysis approach to other two known shape descriptors in the literature, based on shape histograms and shape distribution.

Thus, this paper starts describing how shape complexity analysis is performed in 3D models using a multi-scale approach (Section 2). In Section 3, we describe the experiments performed on the 3D model data base 9] (available at http://segeval.cs.princeton.edu) and the compared methods. Results are presented and discussed in Section 4, while Section 5] describes the conclusions drawn from this work.

\section{Fractal Dimension Based Approach}

Some of the literature on object descriptors define the fractal dimension as an interesting parameter to characterize roughness in an object [10]. Among the many approaches developed to estimate this parameter, Bouligand-Minkowski method [5]11]12] has emerged as the one that presents the most accurate results and has shown to be very sensitive to structural changes of the object. This method was originally developed for shape analysis. However, recent studies have described its use in texture analysis, an evidence that the method can also be effective in estimating the complexity of 3D models [613].

Let $S=\left[s_{1}, s_{2}, \ldots, s_{N}\right]$, be the set of vertices that compose the faces of a 3D model. Each vertex $s_{i}$ is defined by the triple $(x, y, z)$, where $x, y$ and $z$ are the coordinates of a vertex in $R^{3}$. Bouligand-Minkowski method is based on the study of the influence volume of an object computed from its dilation. Thus, let $V(r)$ be the dilation of $S$ by a sphere of radius $r$ :

$$
V(r)=\left\{s_{j} \in R^{3}\left|\exists s_{i} \in S:\right| s_{i}-s_{j} \mid \leq r\right\},
$$

where $s_{j}=\left(x_{j}, y_{j}, z_{j}\right)$ is a point in $R^{3}$ whose distance from $s_{i}=\left(x_{i}, y_{i}, z_{i}\right)$ is smaller or equal to $r$. Figure 1 shows an example of this dilation process.

From the study of the influence volume, the fractal dimension $D$ can be estimated as

$$
D=3-\lim _{r \rightarrow 0} \frac{\log V(r)}{\log (r)},
$$

where $D$ is a number within $[0 ; 3]$ related to the roughness of the shape. 


\begin{tabular}{|c|c|c|}
\hline$x$ & $y$ & $z$ \\
\hline 1 & 1 & 9 \\
\hline 1 & 2 & 29 \\
\hline 1 & 3 & 19 \\
\hline 1 & 4 & 13 \\
\hline 2 & 1 & 1 \\
\hline 2 & 2 & 17 \\
\hline 2 & 3 & 22 \\
\hline 2 & 4 & 23 \\
\hline 3 & 1 & 21 \\
\hline 3 & 2 & 12 \\
\hline 3 & 3 & 11 \\
\hline 3 & 4 & 24 \\
\hline 4 & 1 & 20 \\
\hline 4 & 2 & 6 \\
\hline 4 & 3 & 0 \\
\hline 4 & 4 & 28 \\
\hline
\end{tabular}

(a)

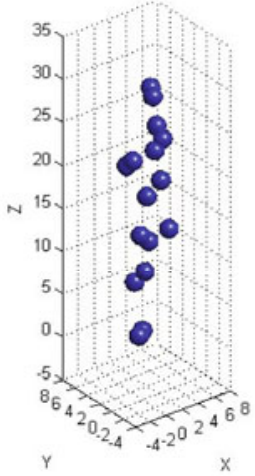

(b)

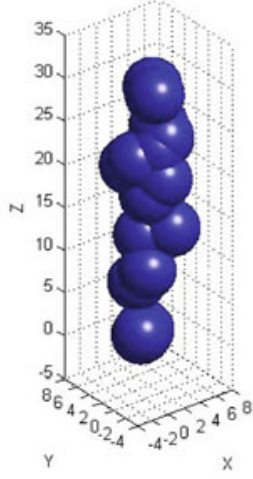

(c)

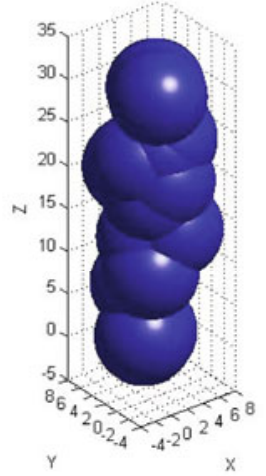

(d)

Fig. 1. Example of the influence volume $V(r)$ computed for a set of points in (a); (b)-(d) Influence volume for different radius values $(r=\{1,3,5\})$. As the radius increases, more interaction among the spheres occurs, thus producing an influence volume characteristic for the set of points.

Regardless of the large sensitiveness of the method to structural changes, there are cases where a single non-integer value is not sufficient to describe all levels of detail present in the influence volume $V(r)$ for a specific object. A better description of the 3D model, in terms of its complexity, is achieved by using the concept of Multi-Scale Fractal Dimension [1415]11]. Different from linear interpolation, which is commonly used to compute the angular coefficient of the log-log curve $r \times V(r)$, this approach exploits the infinitesimal limit of the linear interpolation by using the derivative of the log-log curve. As a result, a function that expresses the complexity of the object in terms of the spatial scale is yielded (Figure 2). This function enables us to perform a more effective discrimination of the object, and it is defined as:

$$
D(r)=3-\frac{d \log V(r)}{d r},
$$

where $D(r)$ represents the complexity of the object at scale $r$.

\section{Evaluation of the Shape Descriptor}

In order to evaluate the proposed approach, an experiment was performed using a set of artificial 3D models. This set comprises $3803 \mathrm{D}$ models grouped into 19 different classes with 20 samples each. Each class is composed by different orientations as also variations (e.g., articulation) of a given shape structure. Figure 3 shows some examples of $3 \mathrm{D}$ models in the data set [9] (available at http://segeval.cs.princeton.edu).

Multi-scale fractal dimension curves were computed from each model. The technique was set up considering a dilation radius $r=20$, and the derivative was 


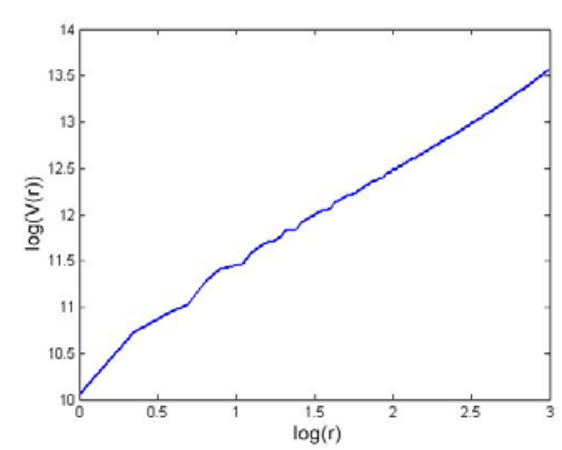

(a)

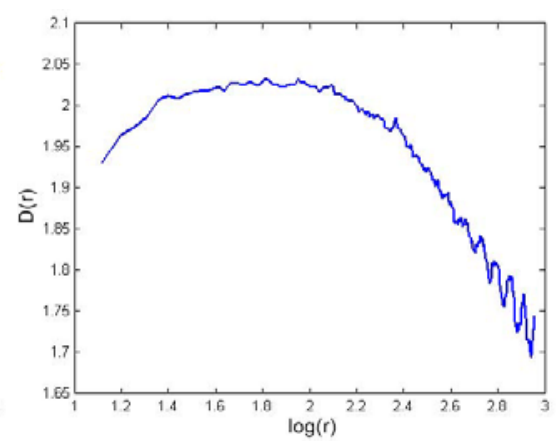

(b)

Fig. 2. (a) Log-log curve computed from shape dilation; (b) Multi-scale Fractal Dimension

computed using the Finite Difference method [16. Resulting curves were evaluated using Linear Discriminant Analysis (LDA), a supervised statistical classification method 17/18, which searches for a linear combination of the descriptors that results in its class. The main goal of the method is to find a linear combination that maximizes the intra-class variance while minimizes the inter-class variance. The leave-one-out cross-validation strategy was also used over the LDA.

\subsection{Compared Approaches}

This new formulation was implemented and compared to two known approaches, also implemented, for 3D shape description and matching. The compared approaches are: (i) 3D shape histograms and (ii) Shape distributions. These are statistical properties methods and they were selected as our approach resembles them, since it computes the distribution of influence volume for a specific dilatation radius. A brief description about those approaches is given below.

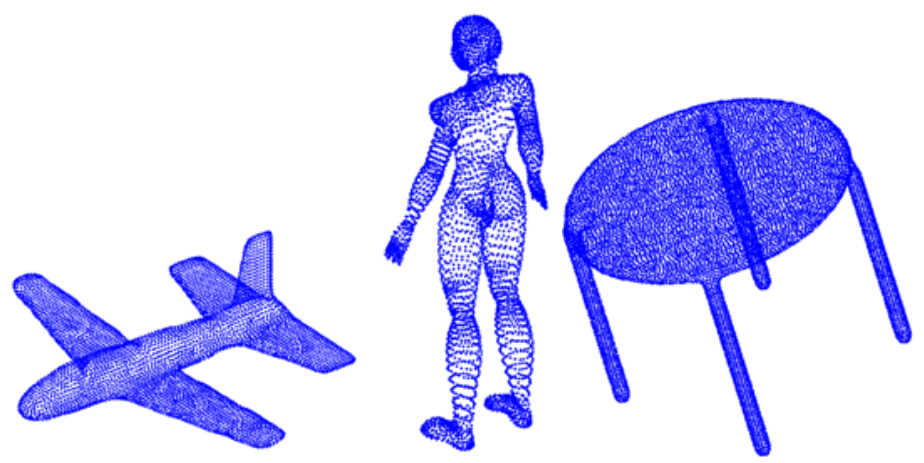

Fig. 3. Examples of 3D shapes used in the experiments 

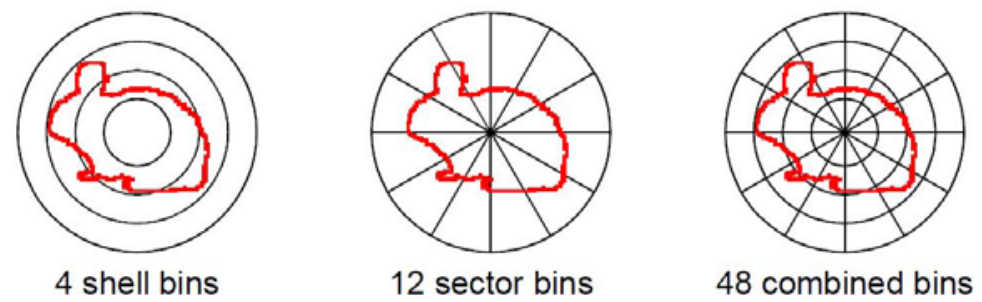

Fig. 4. Space decomposition based on shells, sectors and both combined. Each partition is a single bin in the resulting histogram, and each bin represents the amount of shape in that partition. Adapted from [19].

3D shape histograms: Ankerst et al [19] proposed an approach to compute discrete representations from 3D objects based on 3D shape histograms. In this approach, the space is decomposed based on one of the three suggested techniques: shell model, sector model and the combination of the previous techniques, as shown in Figure 4 . The histogram computation is performed with the chosen space decomposition technique, and each bin stores the amount of vertices positioned in each partition of the decomposed space.

To avoid translation and rotation problems, before the histogram computation a normalization step is applied over the 3D object. Firstly, the center of mass of the objects is placed onto the origin. Then, a $3 \times 3$ covariance matrix is computed over the coordinates of all points from which the eigenvectors, representing the principal axes of the original 3D object, and eigenvalues, indicate the variance of the points in the respective eigenvector direction, are achieved. Finally, the Principal Axes Transform [19] is performed over the 3D object.

Shape distributions: Osada et al. [20] proposed a method to describe 3D objects as a probability distribution sampled from the object as a shape function, which reflects geometric properties of the object. The method computes histograms, known as shape distributions, using a specific measure computed from randomly selected points on the surface of the $3 \mathrm{D}$ object.

To achieve good classification and retrieval results, it is essential to select a shape function whose distribution provides a good shape signature. Osada et al. 20. presented some experiments using distinct shape functions. The best results were achieved using the D2 shape function, which represents the shape of a 3D model by the distribution of Euclidean distances between pairs of points on its surface.

\section{Results}

Table 1 shows classification results yielded for the proposed and the compared approaches. Multi-scale fractal dimension curves were computed from each 3D object considered. The best results for this set of artificial 3D models were yielded considering dilation radius $r=20$. Derivative was computed using the Finite 
Table 1. Comparison of the success rates for different shape descriptors

\begin{tabular}{|lcc|}
\hline Method & Objects correctly classified Success rate $(\%)$ \\
\hline 3D shape histogram & 165 & 43.42 \\
$\begin{array}{l}\text { Shape distribution } \\
\text { Shape complexity analysis } \\
\text { (proposed approach) }\end{array}$ & 256 & 67.37 \\
\hline
\end{tabular}

Difference method [16], and the 130 first points of the curve were considered as descriptors of the $3 \mathrm{D}$ object.

For the 3D shape histogram, a histogram based on 10 shells and 8 sectors was considered instead of those proposed in the original paper $(20$ sectors and 6 or 12 shells). This configuration was preferred as it leads to a higher success rate $(43.42 \%)$ than the one achieved for the other configurations $(38.42 \%$, for both 6 or 12 shells) for this set of artificial 3D models. For the dissimilarity of the shape distributions, it was measured based on L1 norm of the probability density function of the D2 shape function. A normalization step was performed by aligning the mean sample values of two compared probability density functions.

Results show that the proposed approach surpasses the effectiveness of the compared ones, as it is more robust in the classification of the 3D models evaluated. This is mostly due to the great sensitiveness and accuracy of the BouligandMinkowski method to detect small changes in the surface model. Moreover, the approach is invariant to rotation, i.e., no previous normalization step is necessary during its calculus, due to the use of the Euclidean distance. The main disadvantage of the method lies in the dilatation process, which is performed in a discrete space using the Euclidean Distance Transform (EDT) 2122. Since the original $3 \mathrm{D}$ model data was defined at interval $[0,1]$, it was necessary to normalize it to $[0,50]$ before the dilatation process took place.

Note that the shape distribution approach is also invariant to rotation. As this approach uses random sampling during calculations, it is also insensitive to small perturbations in the object (e.g., articulation). These characteristics of the method explain its performance.

The inefficient discrimination achieved by the 3D shape histogram is mostly due to the presence of articulation in some 3D models. Although, this method uses a normalization step to avoid rotation invariance and a quadratic form distance function as an adaptable similarity function, the presence of articulations and others small variations results in the same basic structure, for two different models, mapped in different bins of the histogram and, therefore, does not reflect the close similarity of the models.

\section{Conclusions}

This paper proposes a novel approach to discriminate $3 \mathrm{D}$ models based on shape's complexity analysis methods. Multi-scale fractal dimension curves are used to represent the 3D shape. These curves are computed based on the space occupation and self-similarity of the $3 \mathrm{D}$ shape. 
The proposed approach was evaluated using a 3D model data set 9] and reported interesting results to discriminate different 3D models, significantly surpassing two other known methods: 3D Shape Histograms and Shape Distributions. The compared approaches are not sensible enough to make subtle distinctions. Whereas our approach improved the effectiveness and the results also demonstrated its robustness in dealing with different shape, rotation and articulation of 3D models. Furthermore, the parameterization of our approach is simpler than the compared.

In the future, we plan to perform new experiments in a larger data set (e.g., The Princeton Shape Benchmark [23]) and to compare our approach to other classes of shape discriminator methods.

\section{Ackowledgements}

A.R.B. acknowledges support from FAPESP (2006/54367-9). D.M.E. acknowledges support from FAPESP (2007/04241-1). R.M acknowledges support from CNPq (301295/2008-5). O.M.B. acknowledges support from CNPq (306628/ 2007-4).

\section{References}

1. Osada, R., Funkhouser, T., Chazelle, B., Dobkin, D.: Matching 3D models with shape distributions. In: SMI 2001: Proceedings of the International Conference on Shape Modeling \& Applications, Washington, DC, USA, p. 154. IEEE Computer Society, Los Alamitos (2001)

2. Yang, Y., Lin, H., Zhang, Y.: Content-based 3-D model retrieval: A survey. IEEE Transactions on Systems, Man, and Cybernetics 37(6), 1081-1098 (2007)

3. Bimbo, A.D., Pala, P.: Content-based retrieval of 3d models. ACM Trans. Multimedia Comput. Commun. Appl. 2(1), 20-43 (2006)

4. Tangelder, J.W., Veltkamp, R.C.: A survey of content based 3d shape retrieval methods. Multimedia Tools Appl. 39(3), 441-471 (2008)

5. Tricot, C.: Curves and Fractal Dimension. Springer, Heidelberg (1995)

6. Backes, A.R., Casanova, D., Bruno, O.M.: Plant leaf identification based on volumetric fractal dimension. IJPRAI 23(6), 1145-1160 (2009)

7. da Costa, L.F., Cesar Jr., R.M.: Shape Analysis and Classification: Theory and Practice. CRC Press, Boca Raton (2000)

8. Carlin, M.: Measuring the complexity of non-fractal shapes by a fractal method. PRL: Pattern Recognition Letters 21(11), 1013-1017 (2000)

9. Chen, X., Golovinskiy, A., Funkhouser, T.: A benchmark for 3D mesh segmentation. ACM Transactions on Graphics (Proc. SIGGRAPH) 28(3) (2009)

10. Sarkar, N., Chaudhuri, B.B.: An efficient approach to estimate fractal dimension of textural images. Pattern Recognition 25(9), 1035-1041 (1992)

11. de Plotze, R.O., Falvo, M., Pádua, J.G., Bernacci, L.C., Vieira, M.L.C., Oliveira, G.C.X., Bruno, O.M.: Leaf shape analysis using the multiscale Minkowski fractal dimension, a new morphometric method: a study with passiflora (passifloraceae). Canadian Journal of Botany 83(3), 287-301 (2005)

12. Bruno, O.M., de Plotze, R.O., Falvo, M., de Castro, M.: Fractal dimension applied to plant identification. Information Sciences 178, 2722-2733 (2008) 
13. Backes, A.R., de Sa Jr., J.J.M., Kolb, R.M., Bruno, O.M.: Plant species identification using multi-scale fractal dimension applied to images of adaxial surface epidermis. In: Jiang, X., Petkov, N. (eds.) CAIP 2009. LNCS, vol. 5702, pp. 680-688. Springer, Heidelberg (2009)

14. Emerson, C.W., Lam, N.N., Quattrochi, D.A.: Multi-scale fractal analysis of image texture and patterns. Photogrammetric Engineering and Remote Sensing 65(1), 51-62 (1999)

15. Gonzalez, R.C., Woods, R.E.: Digital Image Processing, 2nd edn. Prentic-Hall, New Jersey (2002)

16. Smith, G.D.: Numerical Solution of Partial Differential Equations: Finite Difference Methods, 3rd edn., Oxford (1986)

17. Everitt, B.S., Dunn, G.: Applied Multivariate Analysis, 2nd edn. Arnold, London (2001)

18. Fukunaga, K.: Introduction to Statistical Pattern Recognition, 2nd edn. Academic Press, London (1990)

19. Ankerst, M., Kastenmüller, G., Kriegel, H.P., Seidl, T.: 3D shape histograms for similarity search and classification in spatial databases. In: Güting, R.H., Papadias, D., Lochovsky, F.H. (eds.) SSD 1999. LNCS, vol. 1651, pp. 207-226. Springer, Heidelberg (1999)

20. Osada, R., Funkhouser, T., Chazelle, B., Dobkin, D.: Shape distributions. ACM Transactions on Graphics 21(4), 807-832 (2002)

21. Bruno, O.M., da Fontoura Costa, L.: A parallel implementation of exact Euclidean distance transform based on exact dilations. Microprocessors and Microsystems 28(3), 107-113 (2004)

22. Fabbri, R., da Fontoura Costa, L., Torelli, J.C., Bruno, O.M.: 2D Euclidean distance transform algorithms: A comparative survey. ACM Computing Surveys 40(1), 1-44 (2008)

23. Shilane, P., Min, P., Kazhdan, M., Funkhouser, T.: The Princeton shape benchmark. In: SMI 2004: Proceedings of the Shape Modeling International 2004, Washington, DC, USA, pp. 167-178. IEEE Computer Society, Los Alamitos (2004) 